\title{
Changes in daily life reduce indoor exposure to selected endocrine disruptors in the home environment: a pilot intervention study*
}

\author{
Aleksandra Rutkowska1,2, Aleksandra Olsson², Monika Piotrowska-Szypryt ${ }^{4}$ and \\ Jacek Namieśnik ${ }^{3+}$
}

1Department of Nursing Management, Medical University of Gdansk, Gdańsk, Poland; ${ }^{2}$ DetoxED Ltd., Gdańsk, Poland; ${ }^{3}$ Department of Analytical Chemistry, Faculty of Chemistry, Gdansk University of Technology, Gdańsk, Poland; ${ }^{4}$ Gdansk Water Utilities Ltd., Gdańsk, Poland

Increasing prevalence of lifestyle diseases raised global awareness about health consequences of human exposure to endocrine disruptors (EDs): synthetic chemicals that mimic natural hormones and affect the biochemical and endocrine balance. As home environment is one of the main sources of the exposure to xenobiotics - especially for pregnant women, infants and young children health organizations emphasize the need of implementing lifestyle changes to protect human health and child development. The aim of this study was to evaluate the effectiveness of introducing changes in daily life in lowering the exposure to selected EDs in the indoor home environment. Twenty-six healthy volunteers from 9 households from Gdansk (Poland) were enrolled and their home- and lifestyle-related exposure to EDs was analyzed using a designed questionnaire and algorithm. Urine and dust samples were collected before and after introducing the recommended lifestyle changes. The concentrations of selected EDs in the samples were determined using liquid chromatography-electrospray ionization tandem mass spectrometry (LC-ESI-MS/MS). This pilot study confirmed the ubiquity of harmful chemicals in the home environment and the importance of exposure related to a daily routine. Importantly, it proved that lifestyle modifications implemented by participants led to a significant decrease in both, their home-related exposure to EDs, as well as in urine concentrations of these chemicals. It also demonstrated a need for determining EDs exposure and introducing lifestyle changes as a useful tool for prevention of lifestyle-related diseases.

Key words: biomonitoring, bisphenol, endocrine disruptors, indoor environment, phthalates, lifestyle diseases

Received: 15 May, 2020; revised: 25 May, 2020; accepted: 26 May, 2020; available on-line: 19 June, 2020

অe-mail: aleksandra.rutkowska@gumed.edu.pl

tDeceased 14 April 2019

Acknowledgements of Financial Support: The intervention and chemical analysis of endocrine disruptors were partially supported by the Interreg Baltic Sea Region NonHazCity project: Innovative management solutions for minimizing emissions of hazardous substances from urban areas in the Baltic Sea Region (NonHazCity, \#R010) and Gdansk Water Utilities Ltd.

*These results were presented as a report during a meeting of partners involved in the European Interreg Baltic Sea Region (NonHazCity) project.

Abbreviations: BPA, bisphenol A; BPS, bisphenol S; DEP, diethyl phthalate; DiBP, diisobutyl phthalate; DEHP, bis(2-ethylhexyl)phthalate; EDs, endocrine disruptors; 4-NP, nonylphenol; LC-ESI-MS/MS, liquid chromatography-electrospray ionization tandem mass spectrometry; LOD, limit of detection

\section{INTRODUCTION}

Increasing prevalence of lifestyle-related diseases raised global awareness about health consequences of human exposure to the so-called endocrine disruptors (EDs), and has recently become of great interest. EDs, similar in chemical structure to hormones, affect their proper functioning; thus, an exposure to their action (especially in the prenatal and early-life) may lead to increased risks of endocrinopathies, cancers, obesity, other metabolic and fertility disorders, preterm birth, developmental disorders, and epigenetic changes in subsequent generations (Gore et al., 2015). Examples of chemical structures of some EDs and natural estrogens are shown in Fig. 1.

Currently, almost 1,500 chemicals (e.g. pesticides, plasticizers, and industrial intermediates) are recognized as EDs and the number is continuously growing (ECHA et al., 2018). Some are used as additives in plastic, such as bisphenol A (BPA) and its derivatives, 4-nonylphenol (4$\mathrm{NP}$ ), and phthalates. Hence, they are commonly used in food packaging, plastic bottles, electronics, toys, accessories for children, cosmetics, cleaning agents, textiles, paints, polyvinyl chloride products, furniture, carpets, receipts, and many others (Konieczna et al., 2015; Wu et al., 2018). Cumulative effect of lifestyle, indoor and environmental pollution leads to constant EDs exposure (transdermally, by inhalation, and ingestion), which may have an impact on increasing a risk of hormonal, biochemical, and metabolic disorders (Rutkowska et al., 2020). As home environment is one of the main sources

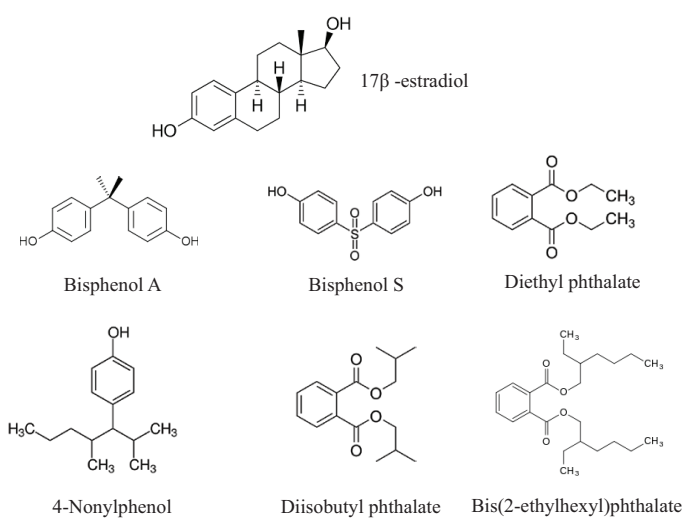

Figure 1. Chemical structure of endocrine disruptors (EDs) studied and estrogen - 17b-estradiol.

The similarity is seen in phenolic ring moiety; thus, such EDs are commonly called xenoestrogens. 
of the exposure - especially for pregnant women, infants and young children - health organizations emphasize the need of implementing lifestyle changes to protect human health and child development (WHO-UNEP, 2012).

Therefore, the aim of this research was to evaluate the effectiveness of introducing changes in daily life in lowering the exposure to selected EDs in the indoor home environment.

\section{MATERIALS AND METHODS}

Volunteers, sample collection and lifestyle changes. This research was a part of a "Gdansk - the city on detox!" campaign of the European Interreg Baltic Sea Region (NonHazCity) project. Twenty-six healthy volunteers (14 males and 12 females) from 9 households from Gdansk (Poland) who lived in a house or an apartment that was built or renovated less than 1.5 years prior to the first sample collection, were enrolled in this study. Before the intervention, at least one household member was interviewed using the EDs exposure questionnaire designed by DetoxED Ltd. Participants were questioned about equipment in rooms (e.g. utensils, cans or plastics in the kitchen), cooking techniques used (cooking products in a loose form or in plastic sacks, etc.), and their personal life (work, cosmetics and household cleaning agent usage, medical procedures, and physical activity). Then, a designed algorithm converted the answers into points to estimate the exposure to EDs (low, medium or high). Modifiers were used according to the declared frequency of use of some items, as well as type and time since household refurbishment. Volunteers were obliged to report any relevant changes, if occurred.

A social media profile was founded by the campaign representatives, who organized regular meetings for volunteers during the period of 6 months. Participants were given advice about reducing their exposure to selected EDs and possible healthier alternatives to household cleaning products, cosmetics, and food packaging, just to name a few. It is worth noting that volunteers received recommendations only, and were supposed to introduce the changes according to their own concept and possibilities at home.

All participants were instructed about proper urine collection and parents were obliged to collect urine samples from their infants and small children. Approximately $60 \mathrm{~mL}$ of mid-stream morning urine was collected

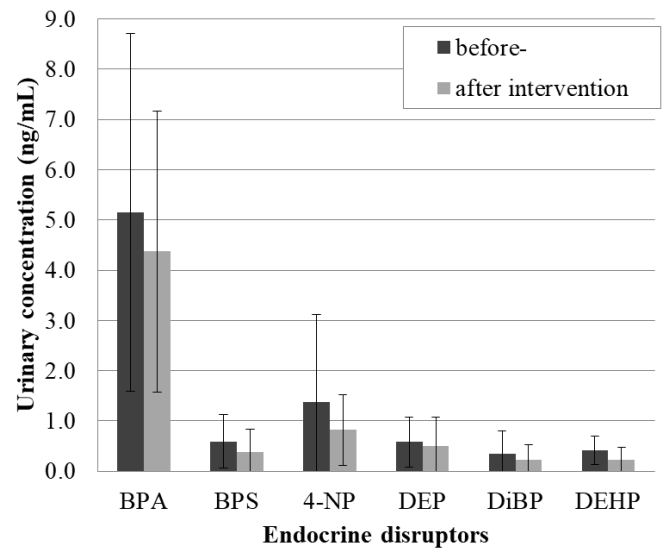

into a glass jar with a metal lid, previously sterilized in autoclave and washed with ethanol.

Volunteers were asked not to clean selected shelves for 3 days prior to dust sampling conducted by DetoxED Ltd. The samples were collected using a cellulose fiber from living room shelves located at a height of 120-150 $\mathrm{cm}$ nearby electronic equipment and then stored in glass vials for analysis. Urine and dust samples were collected first in November 2017 and then in June 2018 - when dust from the floor under the bed was also collected.

Endocrine disruptors analysis in urine and dust samples. The concentrations of six selected EDs in both, the urine and dust (bisphenol A-BPA, bisphenol S - BPS, -nonylphenol-4-NP, DEP - diethyl phthalate, DiBP - diisobutyl phthalate, DEHP - bis(2-ethylhexyl) phthalate), were determined using liquid chromatography-electrospray ionization tandem mass spectrometry (LC-ESI-MS/MS, Shimadzu LCMS-8050, Japan), a method of high sensitivity for quantification of phenolic compounds in urine and environmental samples. All procedures, based on protocols published by (Liao et al., 2012; Guedes-Alonso et al., 2016), were conducted with caution as to not to contaminate the samples with any EDs. Possible contamination from sample processing was evaluated by analysing a reagent blank (distilled water). The limit of detection (LOD) of EDs in urine was $0.5 \mathrm{ng} / \mathrm{mL}$. Values below the LOD were discarded.

Statistics. As it was impossible to conduct the second sampling in two households, eventually only results obtained from 7 families $(n=22)$ that provided samples

Abbreviations: BPA, bisphenol A; BPS, bisphenol S; 4-NP, 4-nonylphenol; DEP, diethyl phthalate; DiBP, diisobutyl phthalate; DEHP, bis(2-ethylhexyl)phthalate; S.D., standard deviation.

Data were statistically analyzed using Statistica 13.3 software (StatSoft, Poland). To verify normal distribution of quantitative data, a Shapiro-Wilk test was performed. The analysis of non-normally distributed variables was performed using non-parametric tests (Mann Whitney U test, Wilcoxon pairs order test). The $P$-value of less than 0.05 was considered as statistically significant.

\section{RESULTS}

Quantifiable EDs levels were found in $100 \%$ of urine $(n=22)$ and dust $(n=7)$ samples at the beginning of the project. After 6 months from implementing lifestyle

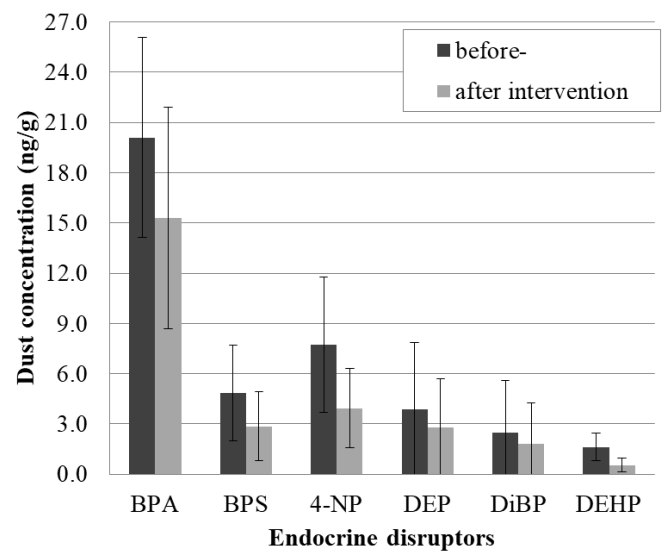

Figure 2. Comparison of the mean (SD) concentrations of selected endocrine disruptors in the urine and dust samples of participants in the "Gdańsk - the city on detox" pilot project before and after implementing lifestyle modifications.

Abbreviations: BPA, bisphenol A; BPS, bisphenol S; 4-NP, 4-nonylphenol; DEP, diethyl phthalate; DiBP, diisobutyl phthalate; DEHP, bis(2ethylhexyl)phthalate; SD, standard deviation. 
modifications, BPA was still quantified in $100 \%$ of the urine samples; however, BPS was detected only in $68 \%$, $4-\mathrm{NP}$ in $95 \%$, DEP in $91 \%$, DiBP in $59 \%$, and DEHP in $55 \%$ of all samples. Furthermore, urine concentrations of all EDs, except for DiBP, were statistically significantly lower than baseline values, with a $P$-value of 0.003 for BPA, 0.004 for BPS, <0.001 for 4-NP and DEHP, and 0.012 for DEP. Significant decrease in the household dust concentration of BPS $(P=0.042)$, 4-NP $(P=0.017)$, and DEHP $(P=0.017)$ was also observed after the intervention. The results are shown in Fig. 2. No differences between dust EDs concentrations in samples collected from the shelves and from under the bed were observed.

There was a visible correlation between EDs concentrations in household dust and urine of each inhabitant. Moreover it was identified both, before and after the intervention: $\mathrm{BPA}-$ before $\left(\mathrm{r}^{2}=0.638, P<0.0001\right)$ and after $\left(\mathrm{r}^{2}=0.613, P<0.0001\right), 4-\mathrm{NP}-$ before $\left(\mathrm{r}^{2}=0.293, P=0.009\right)$, DEP before $\left(r^{2}=0.251, P=0.017\right)$ and after $\left(r^{2}=0.224\right.$, $P=0.022)$, DiBP after $\left(r^{2}=0.500, P<0.001\right)$, and DEHP $\left(r^{2}=0.836, P<0.0001\right)$ before introducing lifestyle changes.

The questionnaire results revealed that concentrations of BPA, BPS, and 4-NP in dust were higher in households with more electronics or plastic elements used, e.g. kitchen utensils, decorations, and boxes; while phthalate concentrations were higher in dust of households with changed floors, walls painted, and with more furniture made from so-called plywood. Participants with higher EDs urine concentrations were also more likely to use plastic in their kitchens and food packaging. Additionally, the consumption of canned food and beverages in plastic bottles, together with a regular contact with store receipts, were associated with higher BPA concentration in urine.

\section{DISCUSSION}

In our pilot study, we measured urinary EDs concentrations, which reflect recent exposure and EDs presence in participants' home environment by determining EDs dust concentration. Our findings were consistent with the results of other researchers who proved the presence of EDs in most of the urine (Larsson et al., 2014) and dust samples (Wang et al., 2014). Additionally, Larrson and coworkers (Larsson et al., 2017) also revealed correlation between selected EDs concentrations and certain foods and hygiene products (Larsson et al., 2014). However, in our study we not only confirmed the ubiquity of harmful chemicals, but also proved that lifestyle changes undertaken by participants led to a significant decrease in their home-related exposure to EDs and in urine concentrations of these compounds. This is a relevant outcome as the participants, also the youngest children and infants, were constantly exposed to a mixture of EDs that may interact with each other and trigger unpredictable biological effects (Gaudriault et al., 2017). Most of the studies conducted in this area were comparably short-term (Harley et al., 2017) or based only on nutritional interventions (Szybiak et al., 2017) rather than on lifestyle changes.

This study was a part of the European Interreg Baltic Sea Region Program of 18 collaborating partners in the Baltic Sea Region. It aimed to identify and prioritize the sources of hazardous substances and contribute to the development of Chemical Action Plans to reduce their emissions. Considering potential adverse effects of the exposure to human health, including hormonal, biochemical, and metabolic disruptions, it is crucial to introduce lifestyle modifications and reduce contact with
EDs. Additionally, it is beneficial not only to minimize the use of products being a source of harmful chemicals, but also to provide the information: where to find or how to prepare healthier substitutes and solutions that minimize the impact of EDs on human health.

\section{Acknowledgements}

We would like to thank Tonie Wickman from the RISE Research Institutes of Sweden and Heidrun Fammler from the Baltic Environmental Forum Germany. Authors are grateful to Jacek Skarbek, CEO of the Gdansk Water Utilities Ltd and Marek Swiniarski, the leader of the NonHazCity project at the Gdansk Water Utilities company, for research support. We would also like to thank Magda Caban from the University of Gdansk and Małgorzata Drewnowska from the City Hall of Gdansk for additional substantive support during the pilot study implementation.

\section{REFERENCES}

ECHA (European Chemicals Agency) and EFSA (European Food Safety Authority) with the technical support of the Joint Research Centre (JRC), Andersson N, Arena M, Auteri D, Barmaz S, Grignard E, Kienzler A, Lepper P, Lostia AM, Munn S, Parra Morte JM, Pellizzato F, Tarazona J, Terron A, Van der Linden S (2018) Guidance for the identification of endocrine disruptors in the context of Regulations (EU) No 528/2012 and (EC) No 1107/2009. EFS A Journal 16: 5311. https://doi.org/10.2903/j.efsa.2018.5311

Gaudriault P, Mazaud-Guittot S, Lavoue V, Coiffec I, Lesné L, Dejucq-Rainsford N, Scholze M, Kortenkamp A, Bernard Jégou B (2017) Endocrine Disruption in Human Fetal Testis Explants by Individual and Combined Exposures to Selected Pharmaceuticals, Pesticides, and Environmental Pollutants. Environ Health Perspect 125: 087004. https://doi.org/10.1289/EHP1014

Gore AC, Chappell VA, Fenton SE, Flaws JA, Nadal A, Prins GS, Toppari J, Zoeller RT (2015) Executive Summary to EDC-2: The Endocrine Society's Second Scientific Statement on Endocrine-Disrupting Chemicals. Endoor Rev 36: 593-602. http://doi.org/10.1210/ er.2015-1093

Guedes-Alonso R, Ciofi L, Sosa-Ferrera Z, Santana-Rodríguez JJ, del Bubba M, Kabir A, Furton KG (2016) Determination of androgens and progestogens in environmental and biological samples using fabric phase sorptive extraction coupled to ultra-high performance liquid chromatography tandem mass spectrometry. J Chromatogr A. 1437: 116-126. https://doi.org/10.1016/j.chroma.2016.01.077

Harley KG, Kogut K, Madrigal DS, et al. (2016) Reducing phthalate, paraben, and phenol exposure from personal care products in adolescent girls: findings from the HERMOSA Intervention Study. Environ Health Perspect. 124: 1600-1607. https://doi.org/10.1289/ ehp.1510514

Konieczna A, Rutkowska A, Rachon D (2015) Health risk of exposure to Bisphenol A (BPA). Rocz Panstw Zakl Hig 66: 5-11.

Larsson K, Lindh CH, Jonsson BA,

Giovanoulis G, Bibi M, Bottai M, Bergström A, Berglund M (2017) Phthalates, non-phthalate plasticizers and bisphenols in Swedish preschool dust in relation to children's exposure. Environ Int 102: 114-124. https://doi.org/10.1016/j.envint.2017.02.006

Larsson K, Ljung Bjorklund K, Palm B, Wennberg M, Kaj L, Lindh CH, Jönsson BA, Berglund M (2014) Exposure determinants of phthalates, parabens, bisphenol A and triclosan in Swedish mothers and their children. Environ Int 73: 323-333. https://doi. org/10.1016/j.envint.2014.08.014

Liao C, Liu F, Guo Y, Moon H-B, Nakata H, Wu Q, Kannan K (2012) Occurrence of eight bisphenol analogues in indoor dust from the United States and several Asian countries: implications for human exposure. Environ Sci Technol 46: 9138-9145. https://doi. org/10.1021/es302004w

Rutkowska A, Olsson A, Wilczewska K, Łukasz Łaczmański Ł, Kuliczkowska-Płaksej J, Jędrzejuk D, Wasik A, Milewicz A, Pasquali R, Urbanovych A, Namieśnik J (2020) Bisphenol A impacts hormonal profile in patients with polycystic ovary syndrome but not in healthy women. Gynecological and Reproductive Endocrinology and Metabolism (GREM) 1: 43-47

Szybiak A, Rutkowska A, Wilczewska K, Wasik A, Namieśnik J, Rachoń D (2017) Daily diet containing canned products significantly increases serum concentrations of endocrine disruptor bisphenol $\mathrm{A}$ in young women. Pol Arch Intern Med 127: 278-280. https://doi. org/10.20452/pamw.4005 
Wang XK, Tao W, Xu Y, Fengd J, Wanga F (2014) Indoor phthalate concentration and exposure in residential and office buildings in Xi'an, China. Atmospheric Environment 87: 146-152. https://doi. org/10.1016/j.atmosenv.2014.01.018

World Health Organisation United Nations Environment Programme (WHO-UNEP). State of the science of endocrine disrupting chemicals. 2012
Wu LH, Zhang XM, Wang F, Gao CJ, Chen D, Palumbo JR, Guo Y, Zeng EY (2018) Occurrence of bisphenol S in the environment and implications for human exposure: A short review. Sci Total Environ 615: 87-98. https://doi.org/10.1016/j.scitotenv.2017.09.194 